\title{
Influence of nonlinear Froude-Krylov forces on the performance of two wave energy points absorbers
}

\author{
Markel Penalba $^{1}$ (D) Alexis Mérigaud ${ }^{1} \cdot$ Jean-Christophe Gilloteaux $^{2} \cdot$ \\ John V. Ringwood ${ }^{1}$
}

Received: 30 November 2016 / Accepted: 11 May 2017 / Published online: 6 June 2017

(C) Springer International Publishing Switzerland 2017

\begin{abstract}
Nonlinear behaviour of wave energy converters in power production mode can be relevant depending on the seastate, the geometry or the motion of the device. Therefore, mathematical models used to simulate the behaviour of the device may need to include nonlinear effects. This paper studies the impact of modelling nonlinear Froude-Krylov forces by computing the pressure over the instantaneous wetted surface and uses a nonlinear wave theory (the RieneckerFenton's theory) including nonlinear free-surface boundary conditions, to be more consistent with the computation of nonlinear Froude-Krylov forces. First, geometric nonlinearities are studied through the consideration of two heaving point absorbers with different geometrical characteristics: a truncated cylinder with a constant cross-sectional area (CSA) and a sphere with a non-uniform CSA. Then, nonlinearities related to the body dynamics are studied by applying a latching control strategy, which highlights the effect of nonlinear dynamics, showing the necessity to consider nonlinear WEC models as a basis for model-based control design. Results show that the performance of a standard fixed-time latching strategy drops considerably when applying nonlinear dynamics, so the fixed-time latching strategy is modified implementing the adaptive latching strategy. The impact of nonlinear Froude-Krylov forces is demonstrated to be low for devices with a constant CSA, but significant for devices of varying CSA.
\end{abstract}

Markel Penalba

mpenalba@eeng.nuim.ie

1 Centre for Ocean Energy Research, Maynooth University, Maynooth, Co. Kildare, Ireland

2 LHEEA Lab. (ECN/CNRS), Ecole Centrale de Nantes, Nantes, France
Keywords Wave energy - Nonlinear incident waves · Nonlinear Froude-Krylov forces · Adaptive latching control

\section{Introduction}

Interest in ocean renewable energy sources has grown considerably over the last two decades, due to their relatively untapped potential. However, the exploitation of ocean waves has not yet reached its technological maturity.

Mathematical models are essential in the design and optimisation process. The vast majority of the hydrodynamic models for wave energy converters (WECs) are based on linear potential theory models, solving the radiation-diffraction problem in the frequency- [WAMIT (WAMIT Inc. 2013), Aquaplus (Delhommeau 1993) or NEMOH (Babarit and Delhommeau 2015)] or the time-domain [ACHILD3D (Babarit 2010)]. These linear models possess very appealing computational properties, but are based on restrictive assumptions, under which the models are accurate. In such linear models, the fluid is assumed inviscid and incompressible, flow is assumed irrotational and wave and device motion amplitudes are assumed small with respect to its dimensions.

The highest efficiency of wave energy absorption is obtained by WECs operating close to resonance, where the high-efficiency bandwidth is generally narrow. As a consequence, it is highly beneficial to artificially bring the device to resonance. Different strategies have been used in the literature: model predictive control (Cretel et al. 2010; Fusco and Ringwood 2010), latching (Ringwood and Butler 2004; Babarit and Clément 2006), phase and amplitude control (Costa et al. 2010; Hals et al. 2016) or reactive control (Hals et al. 2002).

Bringing the device to resonance increases the motion of the device to maximize power production, breaking the 


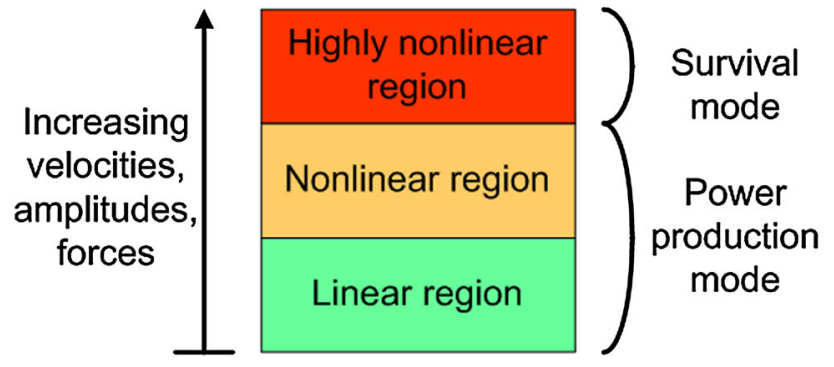

Fig. 1 Different operating regions for wave energy devices

assumptions considered in linear models, which results in significant precision loss. In fact, the inaccuracy of linear models during the survival mode, where high nonlinearities appear, is widely acknowledged. However, it is also possible to meet situations where nonlinear dynamics are significant within the power absorption mode, as Fig. 1 illustrates.

Different studies have shown evidence of the impact of nonlinear dynamics. Penalba et al. (2017) presents the relevance of different nonlinear forces, including FroudeKrylov (FK) forces, and different modelling techniques to consider such nonlinear effects. Discrepancies between linear simulations and experimental tests or nonlinear simulations have been shown, for example, in Babarit et al. (2009) and Merigaud et al. (2012), respectively. Zurkinden et al. (2014) presents a model where nonlinear restoring force is considered, Lawson et al. (2014) and Penalba et al. (2015) describe hydrodynamic models where nonlinear static and dynamic Froude-Krylov forces are implemented, and Gilloteaux (2007) presents a model to predict large amplitude motions by expanding the linear BEM method, considering instantaneous (nonlinear) Froude-Krylov forces and secondorder terms of radiation-diffraction forces.

A previous publication by the same authors, Penalba et al. (2015) had shown the need to combine control strategies and nonlinear computation of hydrodynamic forces. However, the comparison between the cylinder and the sphere presented in Penalba et al. (2015) is biased by the significant difference in the drafts of the devices: the sphere is almost twice as long as the cylinder. Thus, the cylinder exits the water for most of the input wave conditions, showing an important divergence between the linear and nonlinear models, even for the cylinder, which can be misinterpreted.

The present paper studies two geometrically similar devices, a cylinder and a sphere of same radius and draft, using nonlinear waves of reasonable period and amplitude to avoid devices exiting the water. Therefore, the WEC models and set of wave conditions allow for a fair comparison of the effects of nonlinear FK forces for the two devices, when moving freely and under control.

It should be noted that only nonlinear FK forces are implemented, keeping radiation and diffraction forces linear. Thus, the differences observed in this paper between the linear and the nonlinear models can be attributed solely to the nonlinear Froude-Krylov forces in this paper, which is the main focus of the paper. In addition, nonlinear wave theory is used, including nonlinear boundary conditions on the free-surface, which is theoretically more consistent with the implementation of the nonlinear Froude-Krylov forces. Although further research may be necessary, Giorgi et al. (2016) suggests that FK forces are dominant for heaving point absorbers, while Gilloteaux et al. (2008) and Merigaud et al. (2012) suggest that linear approximations for radiation and diffraction can be precise enough within the power production region, also for heaving point absorbers.

It is important to note that, despite the dominance of FK forces over radiation and diffraction for heaving point absorbers, whose length is by definition much smaller than the wavelength, this dominance does not necessarily hold for all the WEC types, where other forces may be dominant, e.g. diffraction and viscous forces appear to be dominant for oscillating surge converters, as suggested by Penalba et al. (2017) and Giorgi et al. (2016).

The present paper is organised as follows: The linear and nonlinear models are described in Sect. 2 and details of the characteristics of the devices and the incident waves are given in Sect. 3. Results are shown in Sect. 4 and discussed in Sect. 5.

\section{Hydrodynamic model formulation}

The hydrodynamic model used in this paper is based on potential theory, where the fluid is inviscid and incompressible and the incident flow is irrotational. The potential of the incident wave can be divided into three parts: undisturbed incident flow, diffracted flow and radiated flow. That way, assuming a device restricted to a single degree of freedom (heave), Newton's law is used to specify the governing equation of a WEC as follows,

$M \ddot{x}=F_{\mathrm{FK}_{\mathrm{st}}}+F_{\mathrm{FK}_{\mathrm{dyn}}}+F_{\mathrm{D}}+F_{\mathrm{R}}+F_{\mathrm{PTO}}$

where $M$ is the mass of the body, $x$ the position of the body relative to its hydrostatic equilibrium and $F_{\mathrm{PTO}}$ the force applied by the power-take-off (PTO) system.

The remainder of the forces are calculated by computing the pressure $P$ of each potential flow acting on the body, using the Bernoulli's equation, where $S$ is the wetted surface of the body and $\vec{n}$ a vector normal to the surface element,

- $F_{\mathrm{FK}}$ is the static Froude-Krylov force, formed by the hydrostatic pressure $\left(P_{\text {stat }}=-\rho g z\right)$ and the gravity force $\left(F_{\mathrm{g}}\right)$ 


$$
F_{\mathrm{FK}_{\mathrm{st}}}=F_{\mathrm{g}}-\iint_{S} P_{\text {stat }} \vec{n} \mathrm{~d} S,
$$

- $F_{\mathrm{FK}_{\mathrm{dyn}}}$ is the dynamic $\mathrm{FK}$ force, computing the dynamic pressure of the incoming wave $\left(P_{\mathrm{dyn}}=-\rho \frac{\partial \Phi_{\mathrm{I}}}{\partial t}-\right.$ $\left.\rho \frac{\left|\nabla \Phi_{\mathrm{I}}\right|^{2}}{2}\right)$, where $\Phi_{\mathrm{I}}$ is the incident potential velocity

$$
F_{\mathrm{FK}_{\mathrm{dyn}}}=-\iint_{S} P_{\mathrm{dyn}} \vec{n} \mathrm{~d} S,
$$

- $F_{\mathrm{d}}$ is the diffraction force $\left(P_{\mathrm{d}}=-\rho \frac{\partial \Phi_{\mathrm{d}}}{\partial t}-\rho \frac{\left|\nabla \Phi_{\mathrm{d}}\right|^{2}}{2}\right)$, where $\Phi_{\mathrm{d}}$ is the diffraction potential velocity

$$
F_{\mathrm{d}}=-\iint_{S} P_{\mathrm{d}} \vec{n} \mathrm{~d} S, \quad \text { and }
$$

- $F_{\mathrm{R}}$ is the radiation force $\left(P_{\mathrm{R}}=-\rho \frac{\partial \Phi_{\mathrm{R}}}{\partial t}-\rho \frac{\left|\nabla \Phi_{\mathrm{R}}\right|^{2}}{2}\right)$, where $\Phi_{\mathrm{R}}$ is the radiation potential velocity

$$
F_{\mathrm{R}}=-\iint_{S} P_{\mathrm{R}} \vec{n} \mathrm{~d} S
$$

Based on Eqs. (2)-(5), linear and nonlinear models can be obtained. Second-order terms in radiation and diffraction potentials are neglected in both, linear and nonlinear models. Since only nonlinear Froude-Krylov forces are considered in the nonlinear model, this nonlinear model is referred to as partially-nonlinear model for the rest of the present paper.

\subsection{Linear model}

In the linear model, free-surface elevation and device motion amplitudes are assumed to be small, so all the quantities of the simulation can be expressed over the mean wetted surface of the floating body.

Following the linear approach, Eq. (1) is written as,

$$
\begin{aligned}
M \ddot{x}= & -K_{\mathrm{H}} x-\int_{-\infty}^{\infty} K_{\mathrm{Ex}}(t-\tau) \eta(\tau) \mathrm{d} \tau \\
& -\mu_{\infty} \ddot{x}-\int_{-\infty}^{\infty} K_{\mathrm{R}}(t-\tau) \dot{x}(\tau) \mathrm{d} \tau-B_{\mathrm{PTO}} \dot{x}
\end{aligned}
$$

where the static Froude-Krylov force is considered to act like a mass-spring system, $K_{\mathrm{H}}$ is the hydrostatic stiffness matrix; the excitation force is formed by the dynamic Froude-Krylov force and the diffraction force and is obtained via the convolution product between the excitation impulse-response $\left(K_{\mathrm{Ex}}\right)$ and the free-surface elevation $(\eta)$; the radiation force is expressed by using the infinite added mass term $\left(\mu_{\infty}\right)$ and a convolution between the radiation impulse-response $\left(K_{\mathrm{R}}\right)$ and the velocity of the body (Cummins 1962); and the power take-off force is modelled as a linear damper using a constant damping coefficient $\left(B_{\mathrm{PTO}}\right)$.

\subsection{Partially-nonlinear model}

The linear approach can be extended by computing the pressure of the potential flow acting on the body over the precise instantaneous wetted surface at each time-step. Two different methods have been found in the literature. The first method uses a very fine mesh that takes into account only those cells of the mesh below the instantaneous free-surface (Babarit et al. 2009). The second method includes an automatic remeshing routine (Gilloteaux et al. 2008), as applied in the framework of this paper. Hence, Eq. (6) can be re-written as,

$$
\begin{aligned}
M \ddot{x}= & F_{g}-\iint_{S(t)}\left(P_{\mathrm{st}}+P_{\mathrm{dyn}}\right) \vec{n} \mathrm{~d} S \\
& -\int_{-\infty}^{\infty} K_{\mathrm{d}}(t-\tau) \eta(\tau) \mathrm{d} \tau \\
& -\mu_{\infty} \ddot{x}-\int_{-\infty}^{\infty} K_{\mathrm{R}}(t-\tau) \dot{x}(\tau) \mathrm{d} \tau-B_{\mathrm{PTO}} \dot{x}
\end{aligned}
$$

where the Froude-Krylov force is integrated over the instantaneous wetted surface and the diffraction force is expressed by the convolution product between the diffraction impulse response $\left(K_{\mathrm{d}}\right)$ and the free-surface elevation $(\eta)$. It should be noted that in the partially-nonlinear case, the diffraction force remains linear as in the linear approach, but it is analysed separately. The same happens with the radiation force and the power take-off force.

\section{Case study}

This paper focuses on the geometric nonlinearities due to variations in the cross-sectional area (CSA) of the devices. To simplify the study, deep-water approximation is assumed, only regular waves are analysed and the devices are restricted to move only in heave. Pressure-related nonlinearities, arising from the exponential decay of dynamic pressure from the free surface to the bottom of the device (where the pressure is applied), can be neglected for point absorbers deployed in deep-water, because the draft of a heaving point absorber is much shallower than the water depth, so that the pressure decay is linear.

Three main aspects are crucial to highlight the effect of geometrical nonlinearities and avoid situations that may lead to misinterpretations of the effect of nonlinear FroudeKrylov forces: the design of the devices, the incident wave conditions and the control strategy. All these aspects are analysed in the following subsections.

\subsection{Body geometries}

Two different shapes are studied: a sphere (which has a nonuniform CSA) and a cylinder (which has a constant CSA), shown in Fig. 2. 
Fig. 2 Sketch view of the two WEC configurations: a sphere and a cylinder
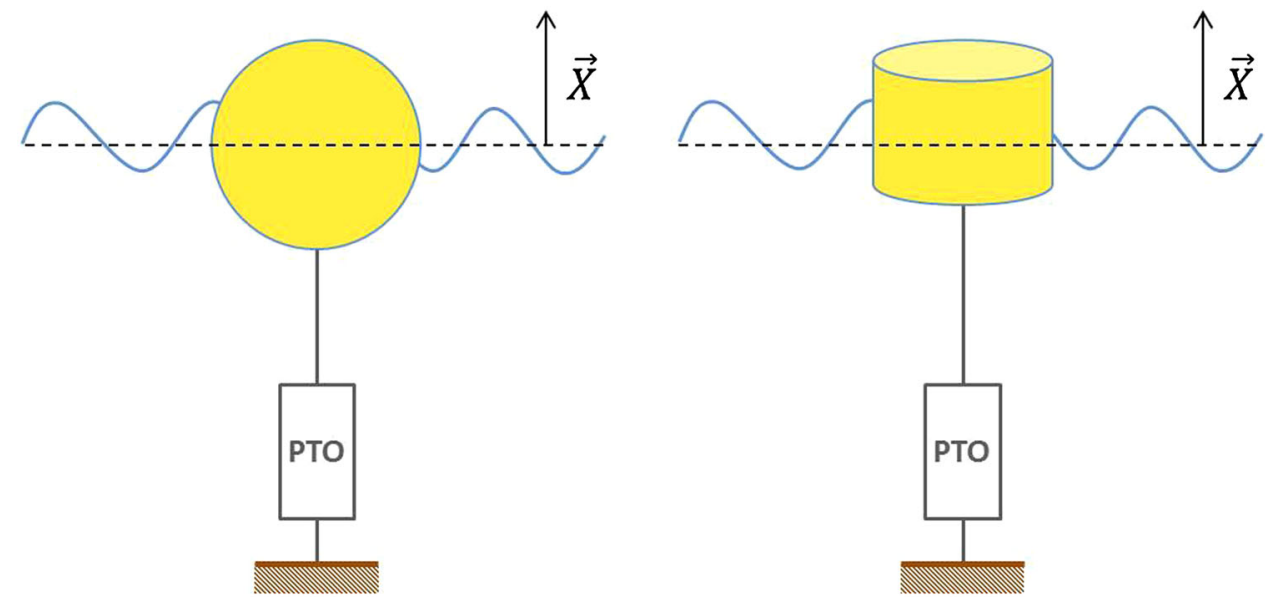

Table 1 Geometrical characteristics of the bodies

\begin{tabular}{lll}
\hline Geometry & Sphere & Cylinder \\
\hline Radius $(\mathrm{m})$ & 2.5 & 2.5 \\
Draft $(\mathrm{m})$ & - & 2.5 \\
Displacement $\left(\mathrm{m}^{3}\right)$ & 32.72 & 49.1 \\
Natural period $(\mathrm{s})$ & 3.2 & 4.1 \\
PTO damping $\left(B_{\text {PTO }}\right)$ & 42,000 & 52,000 \\
\hline
\end{tabular}

In Penalba et al. (2015), the radius and the natural frequency are set equal for the cylinder and the sphere. However, setting natural frequency equal is proven to be irrelevant under control and makes the comparison problematic. Therefore, radius and drafts of the devices are chosen equal, which makes the natural period to be different. Table 1 gives all the details about the two bodies analysed.

\subsection{Incident waves}

Deep-water $\left(\lambda=\frac{g}{2 \pi} T^{2}\right)$ is assumed in the present paper and the steepness value $\left(\frac{H}{\lambda}\right)$ for is set to 0.018 , same as in (Penalba et al. 2015). However, the range of periods studied in the present paper is restricted between 3 and $8 \mathrm{~s}$ to avoid the device to be fully submerged or fully 'dry'. A smaller number of wave periods are considered close to the resonance period of the devices, since those periods are no more relevant than any other (nonzero) wave period when a control strategy that controls the device to resonate with all wave periods is implemented. In addition, the impact of nonlinear FK forces for the shortest wave periods ( 3,4 and $5 \mathrm{~s}$ ) is almost negligible, since the relative water/device displacement is low, as shown in Fig. 5. The wave height increases with the period, but the wave steepness remains constant (0.018) for all the waves. Table 2 shows all the wave periods used in the simulations for this paper, and their corresponding wave heights and wavelengths.
In the previous publication (Penalba et al. 2015), linear wave theory based on Airy's theory shown in Eq. (8) is used,

$\Phi(x, z, t)=\frac{a \omega}{k} \frac{\cosh (k(z+h))}{\sinh (k h)} \sin (k x-\omega t)$

where $\omega$ the wave frequency, $a$ the wave amplitude, $k$ the wavenumber and $h$ the water depth.

However, linear potential analysis relying on Airy's theory, only fulfils the free-surface condition in the first-order $P(z=0)=0$, so that the condition $P(z=\eta)=0$ is not true. Instead of using stretching methods providing predictions of fluid velocity and pressure at points above the mean water level, a nonlinear wave theory is used in the present paper. This nonlinear theory introduces a nonlinear boundary condition on the free-surface, which is more consistent with the use of a partially-nonlinear model to describe Froude-Krylov forces.

\subsubsection{Rienecker-Fenton's theory}

Two main approaches for nonlinear waves have traditionally been used: Stokes' theory and the Cnoidal theory. Stokes' theory appears to be suitable for deep water waves, while the Cnoidal theory seems to be suitable for shallow water waves (Fenton 1990).

Due to the lack of universal applicability of the Stokes' and the Cnoidal theories, a numerical algorithm based on the Fourier approximation technique was created: the Rienecker-Fenton theory (Rienecker and Fenton 1981). The mathematical formulation is based on potential theory and gives the numerical solution of a regular progressive wave.

Rienecker and Fenton present a mathematical formulation using Airy's theory as the first approximation. The Rienecker-Fenton algorithm generates wave-trains for different ocean depths, wave amplitudes and wavelengths, respecting nonlinear limit conditions on the free-surface. 
Table 2 Wave period, height and wavelength of the studied range of wave trains

\begin{tabular}{llllllllll}
\hline Wave periods [s] & 3 & 4 & 5 & 5.5 & 6 & 6.5 & 7 & 7.5 & 8 \\
Wave height [m] & 0.25 & 0.45 & 0.7 & 0.85 & 1 & 1.19 & 1.38 & 1.58 & 1.8 \\
Wavelength [m] & 14 & 25 & 39 & 47 & 56 & 66 & 77 & 88 & 100 \\
\hline
\end{tabular}

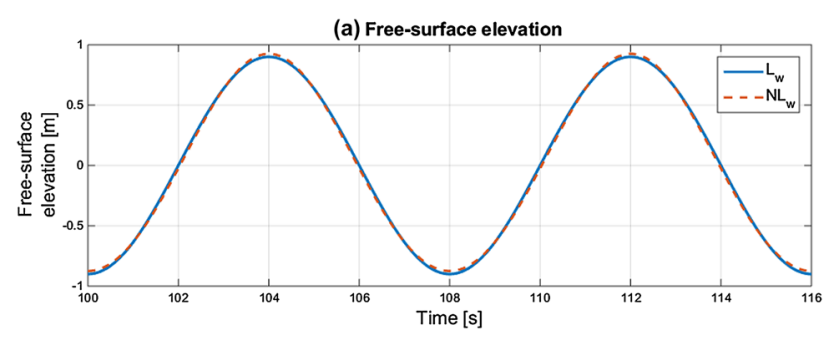

Fig. 3 Free-surface elevation (a) and the corresponding excitation force (b) based on linear and nonlinear wave theories, for a wave of $8 \mathrm{~s}$ of period, computed for the sphere in free motion

$$
\begin{aligned}
& \Phi(x, z, t)=\sum_{i=0}^{n} \Phi_{i}(x, z, t) \\
& \Phi_{i}(x, z, t)=B_{i} \frac{\sinh (i k(z+h))}{\cosh (i k h)} \cos (i k(x-c t))
\end{aligned}
$$

where $B_{i}$ is a coefficient defined in the algorithm presented by Rienecker and Fenton (1981) and $c$ the phase velocity.

Figure 3 illustrates the free-surface elevation for Airy's theory $\left(L_{w}\right)$ and Rienecker-Fenton algorithm $\left(N L_{w}\right)$ for an $8 \mathrm{~s}$ wave computed for the sphere. Due to the low steepness of the incident wave, the free-surface elevation of the linear and nonlinear wave theories appear to be identical. However, the nonlinear wave has a slightly sharper crest and flatter trough, as expected. The reason why a low steepness is chosen is to compare the results with those presented in (Penalba et al. 2015) and find out the relevance of the nonlinear wave theories with nonlinear boundary conditions when implementing nonlinear Froude-Krylov forces.

\subsection{Power absorption and control}

To maximize power absorption for all sea-states, control strategies are used in several studies, adapting the behaviour of the device to each sea-state. In this paper, latching control strategy is applied, which has proven to increase power absorption by a factor of 2 in irregular waves and a factor of 4 in regular waves (Babarit and Clément 2006).

Latching strategy consists of locking (latching) the device when its velocity goes to zero and keeping the device latched until the wave force reaches the most advantageous phase, as shown in Fig. 4, where $\phi$ is the phase offset between the force and the position.

The control variable is the duration of the latching period $\left(T_{\mathrm{L}}\right)$, calculated using the natural period of the device $\left(T_{\omega_{0}}\right)$

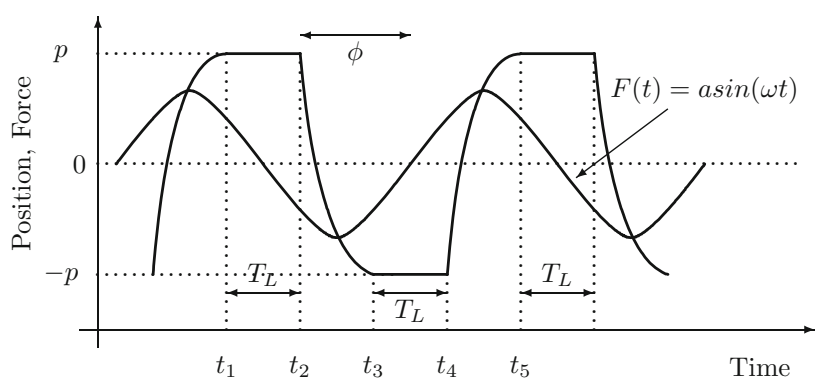

Fig. 4 Latching calculations, (Ringwood and Butler 2004)

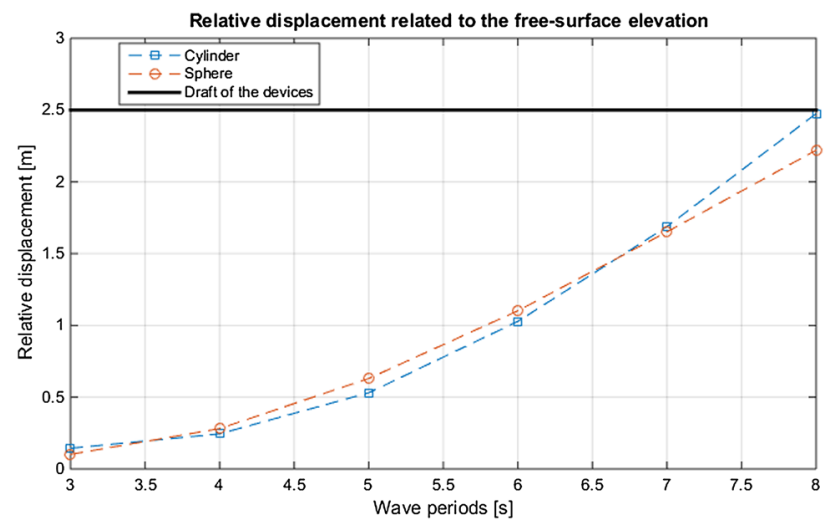

Fig. 5 Device motions relative to the free surface elevation for the cylinder and the sphere compared to the draft of the devices, where device motions are calculated under latching control

and the period of the incident wave $\left(T_{\mathrm{W}}\right)$. Equation (11) shows, to a first approximation, the calculation of the latching period as a function of the timings defined in Fig. 4,

$T_{\mathrm{L}}=\frac{t_{5}-t_{1}}{2}-\left(t_{5}-t_{4}\right)=\frac{T_{\mathrm{W}}}{2}-\frac{T_{\omega_{0}}}{2}$

The PTO system has been modelled as a perfect linear damper, for reasons of simplicity. Table 1 presents the PTO damping value for each device, which is constant for all the different sea-states. Such PTO damping values contribute to avoid the device to be completely submerged or fully 'dry', as shown in Fig. 5, where relative motions between the device motions and the free-surface elevation remain below the limit of the device draft for all the employed sea-states. Figure 5 shows the maximum relative displacement for each wave period.

The nonlinear computation of FK forces, nonetheless, requires the latching strategy to be adapted, as shown in Penalba et al. (2015). Therefore, two strategies have been 


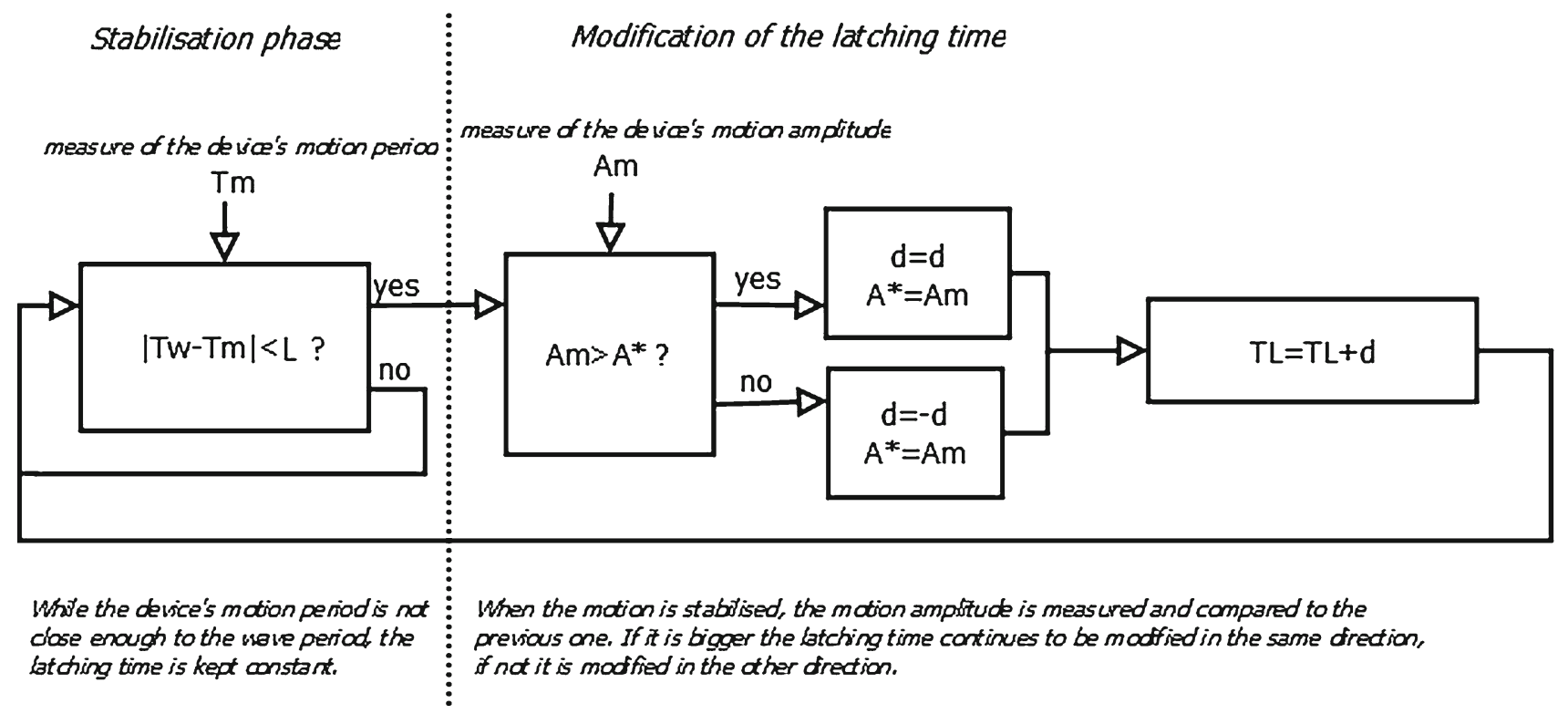

Fig. 6 Diagram of the algorithm the adaptive latching strategy is based on

analysed: fixed-time latching, the optimal strategy for linear computations, and adaptive latching, the adapted strategy for cases where nonlinear FK forces are significant.

\subsubsection{Fixed-time latching}

The fixed-time latching strategy is designed for an optimal performance with linear WEC models, for which the natural period of the device $\left(T_{\omega 0}\right)$ is constant.

\subsubsection{Adaptive latching}

The natural period of the device is not as clearly defined for a model containing nonlinear computation of FK forces, since the hydrostatic stiffness varies significantly for the case where the CSA of the device is not constant.

Therefore, the optimal latching period needs to be adaptively calculated to achieve the greatest motion and power absorption. A basic algorithm presented in Penalba et al. (2015) is used in this paper to identify the optimal latching period.

The adaptive latching algorithm, shown in Fig. 6, consists of modifying the latching period step-by-step in a direction that increases the motion amplitude. Between two consecutive modifications, the algorithm waits for the motion to reach a steady state to get a reliable evaluation of the behaviour of the device. The criterion to confirm steady state has been reached is to check whether the measured period of the device motion $\left(T_{\mathrm{m}}\right)$ is similar to the wave period $\left(T_{\mathrm{W}}\right)$.

In Fig. 6, $L$ is the threshold which determines whether the motion has reached the steady state, $A^{*}$ the memorised motion amplitude obtained with the previous latching time and $d$ the variation of the latching time.
It is important to note that, when applied to the linear hydrodynamic modelling case, the adaptive strategy converges to the fixed-time latching strategy.

\section{Results}

Results are obtained using a Fortran code that performs the time-domain simulation of the WEC motion (using a 4th order Runge-Kutta integration method), able to compute linear and nonlinear Froude-Krylov forces. This Fortran code requires specific time-domain hydrodynamic coefficients and impulse-response functions $\left(K_{\mathrm{H}}, K_{\mathrm{Ex}}\right.$ and $\left.K_{\mathrm{R}}\right)$, which are directly given by the time-domain hydrodynamic code ACHIL3D (Babarit 2010).

A comparison between the linear and the partiallynonlinear models is performed using

- the response amplitude operator (RAO), which illustrates the response of the device over the range of wave periods, and

- the excitation and static FK forces $\left(F_{\mathrm{FK}_{\mathrm{st}}}\right)$.

\subsection{Fixed-time latching strategy}

The fixed-time latching strategy, based on linear models, is applied to the cylinder and the sphere using both the linear and partially-nonlinear hydrodynamic simulation models.

\subsubsection{Cylinder}

The cylinder has a constant CSA, which suggests that geometric nonlinearities may be insignificant. Figure 7 shows 


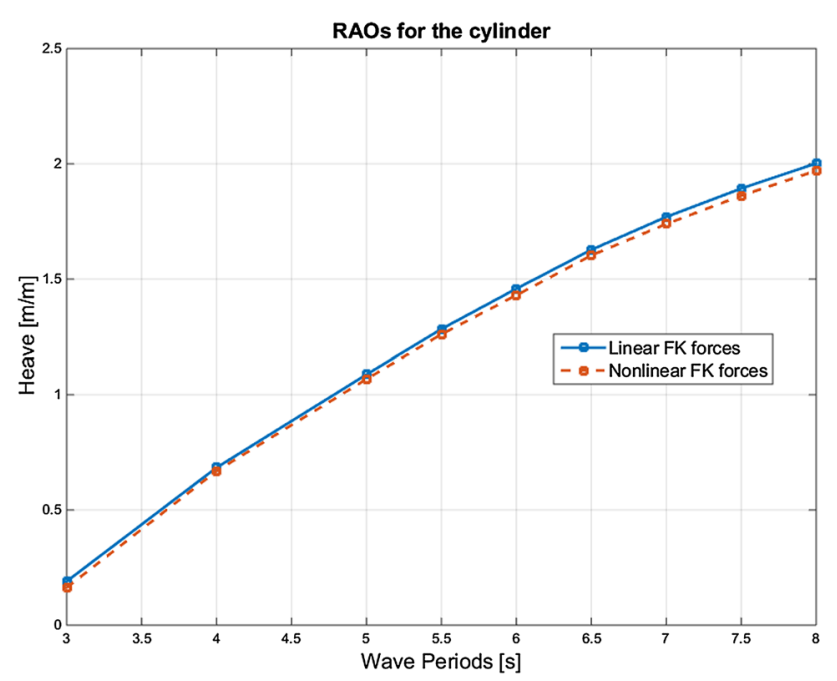

Fig. 7 RAOs for linear and nonlinear computation of FK forces for the cylinder under the fixed-time latching control strategy

RAO values for the linear and partially-nonlinear simulation models, where values for the linear and the partiallynonlinear cases appear to be almost identical.

Figure 8 illustrates the motion and velocity of the cylinder for an $8 \mathrm{~s}$ wave. The amplitude of the motion for the partiallynonlinear case in Fig. 8a is as large as the amplitude of the motion displayed by the linear case. Figure $8 \mathrm{~b}$ shows the device velocity to be perfectly in phase with the excitation force for the linear and partially-nonlinear models, which demonstrates the efficacy of the fixed-time latching strategy for both simulation models.

Static FK forces for the cylinder, shown in Fig. 9, are almost identical for linear and partially-nonlinear models, which confirms that geometric nonlinearities are negligible for the cylinder.

\subsubsection{Sphere}

Unlike in the cylinder case, the partially-nonlinear model has a significant impact in the behaviour of the sphere, due to the non-uniform CSA. The solid and dashed curves in Fig. 10 illustrate RAO values for simulations under the fixed-time latching control, based on the linear and partiallynonlinear models respectively. The difference between linear and nonlinear values under the fixed-time latching increases substantially as wave and device motions become larger. Figure 11a also illustrates the strong reduction of the oscillation amplitude for the partially-nonlinear simulation compared to the linear case, for an $8 \mathrm{~s}$ wave.

The difference between the linear and partially-nonlinear simulations is mostly due to the inability of the fixed-time

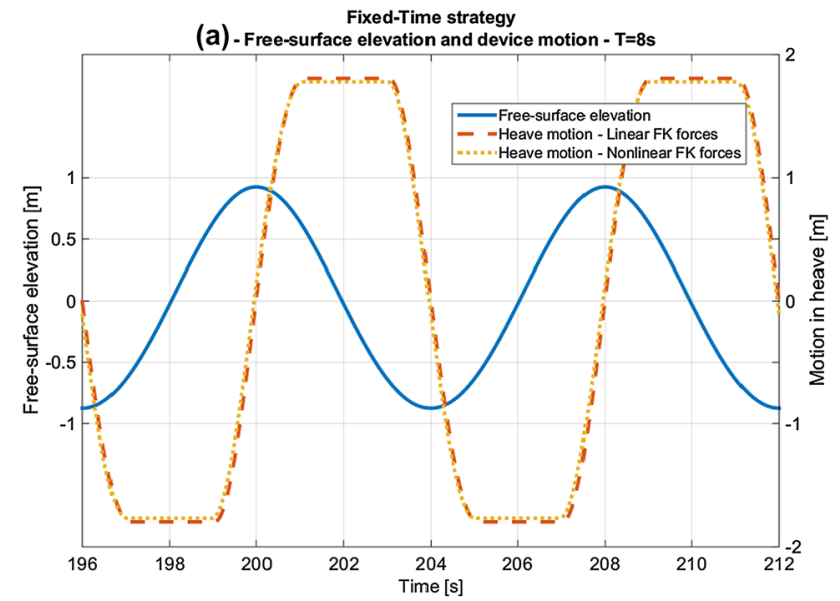

(b) - Free-surface elevation and device velocity $-\mathrm{T}=8 \mathrm{~S}$

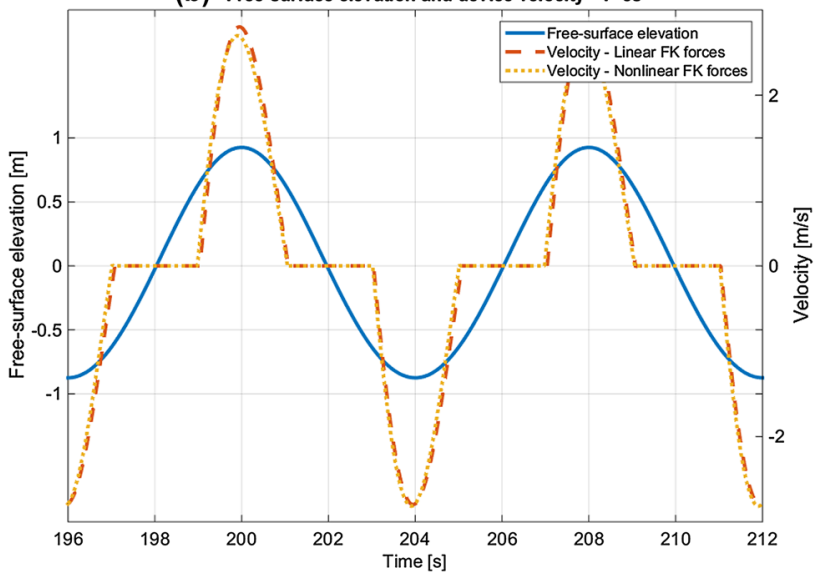

Fig. 8 Motion and velocity of the cylinder for an $8 \mathrm{~s}$ wave and linear and nonlinear computation of the FK forces under the fixed-time latching strategy

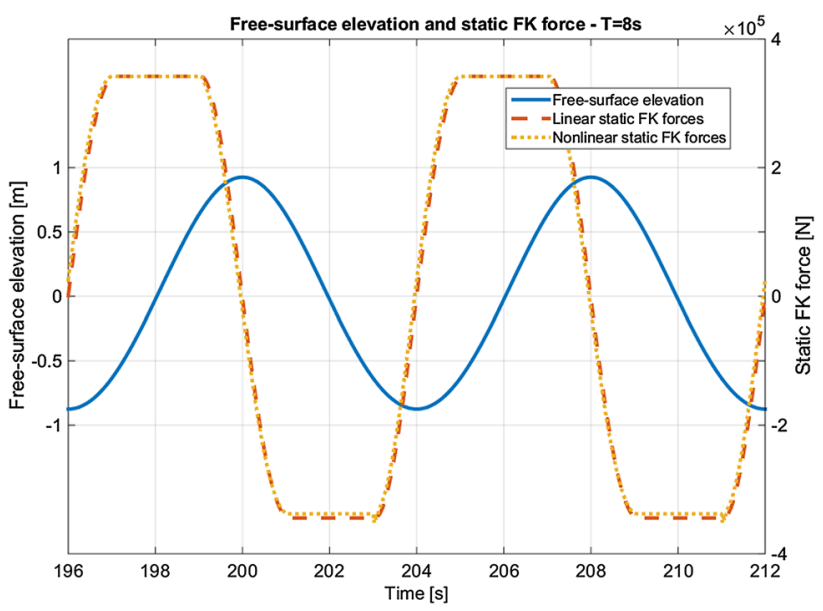

Fig. 9 Static FK forces for an $8 \mathrm{~s}$ wave using the linear and partiallynonlinear simulation models

latching strategy to keep the device velocity and the excitation force in phase in the partially-nonlinear case, as shown in Fig. 11b. 


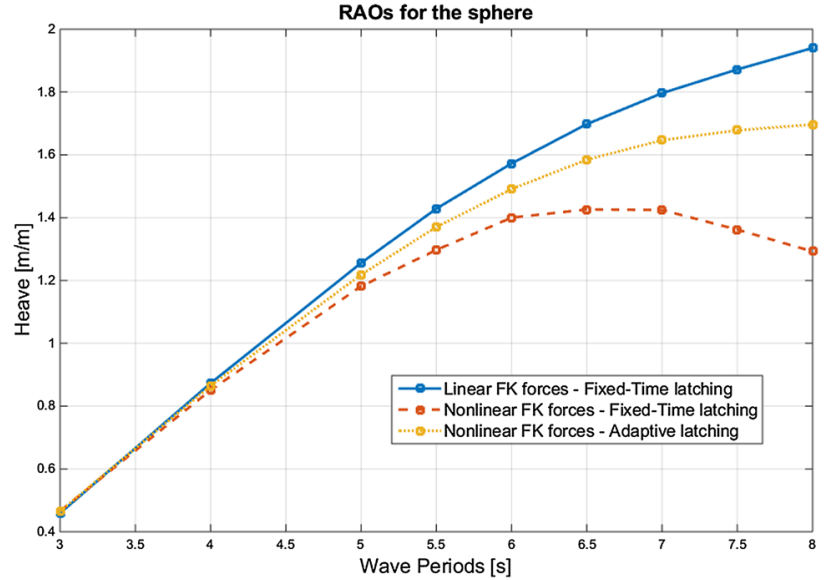

Fig. 10 RAOs for the linear and partially-nonlinear simulation models for the sphere using the fixed-time and adaptive latching control strategies

Hence, when the CSA is not constant, FK force nonlinearities, both static and dynamic, become significant and the performance of the fixed-time latching strategy in partially-nonlinear simulations drops considerably. Therefore, the latching strategy needs to be adapted.

\subsection{Adaptive latching strategy}

An alternative adaptive control strategy is presented in Sect. 3.3.2 based on an algorithm to automatically identify the optimal latching time. This adaptive strategy is only applied to the sphere.

In the presence of significant nonlinearities, the adaptive latching strategy shows the ability to maximise the motion of the device. Figure 11a, c illustrate the performance of the adaptive latching strategy. When applied to the linear model, the adaptive latching strategy provides identical results to the fixed-time latching strategy. In contrast, when applied to the partially-nonlinear model, the adaptive latching strategy substantially enlarges the amplitude of device motion, compared to the case where the fixed-time strategy is applied.

Such improvement in the control performance of the adaptive latching strategy is due to the adjustment of the latching

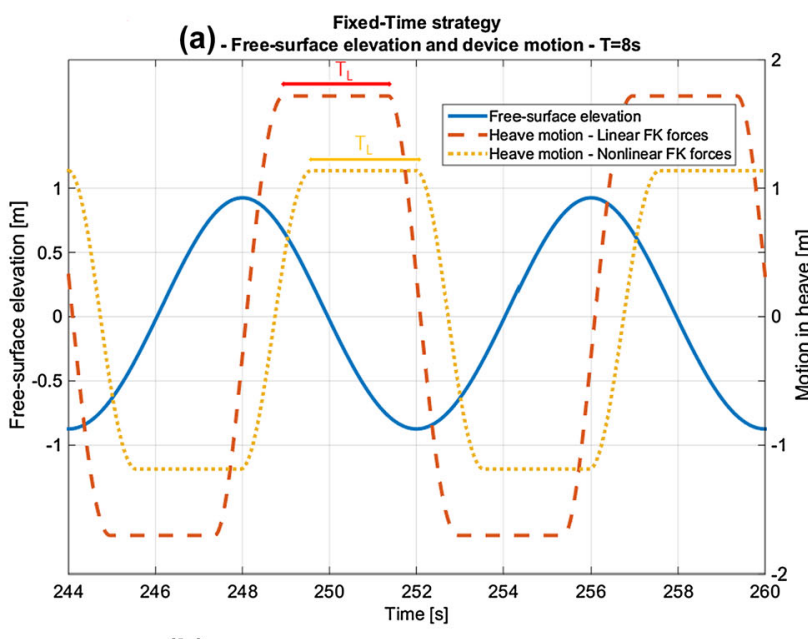

(b) - Free-surface elevation and device velocity $-\mathrm{T}=8 \mathrm{~s}$

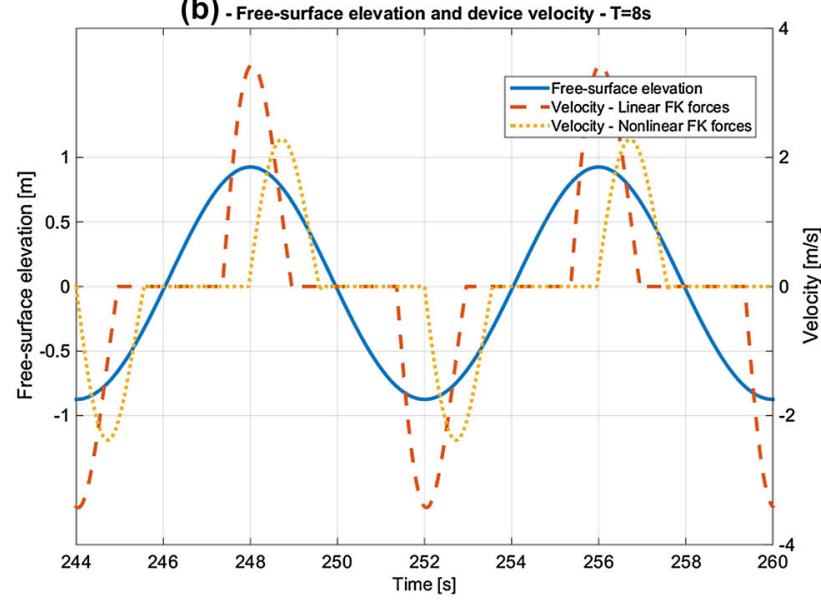

(c) - Free-surfaptive strategy

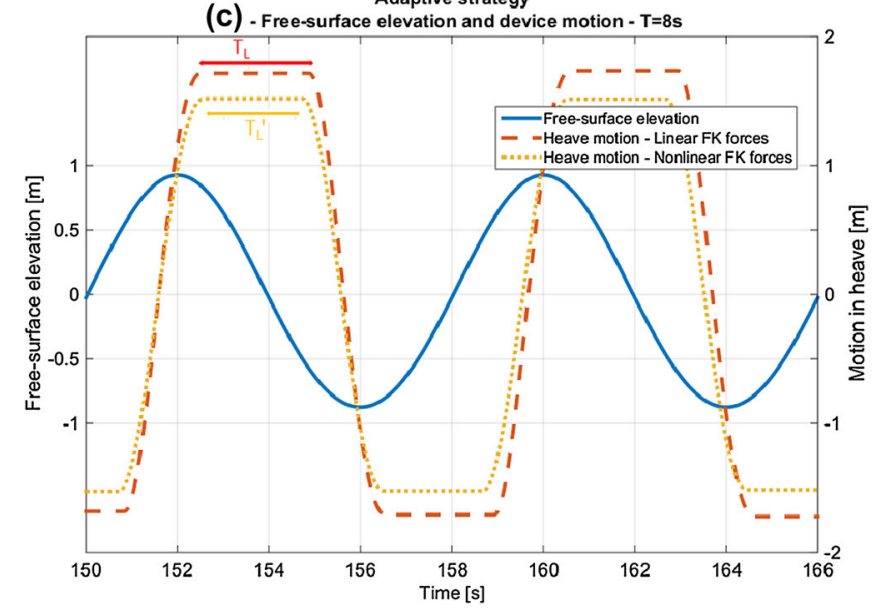

(d) - Free-surface elevation and device velocity - $T=8 \mathrm{~s}$

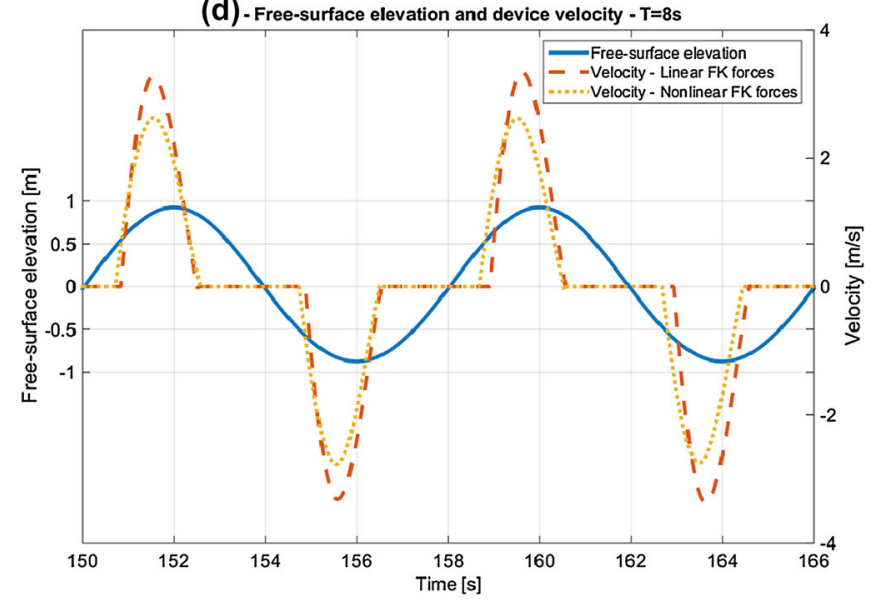

Fig. 11 Free-surface elevation, motion and velocity for the fixed-time latching (left column) and adaptive latching (right column) control strategies for linear and partially-nonlinear simulation models for the $8 \mathrm{~s}$ wave 

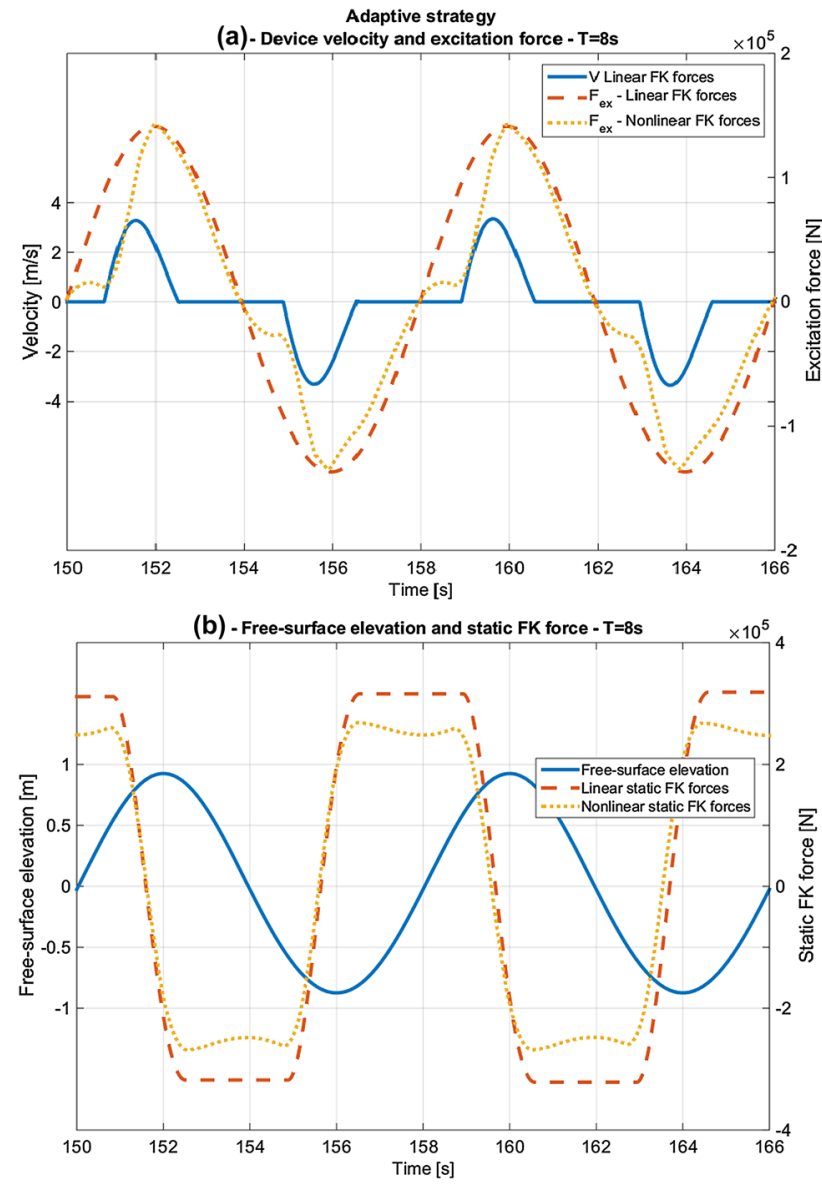

Fig. 12 Excitation and static FK forces under the adaptive latching strategies for linear and partially-nonlinear models for an $8 \mathrm{~s}$ wave

time to the slower nonlinear dynamics of the sphere. The adaptive algorithm calculates the optimal latching time for the partially-nonlinear model $\left(T_{\mathrm{L}}^{\prime}\right)$, which is shorter than the optimal latching time for the linear case $\left(T_{\mathrm{L}}\right)$, as shown in Fig. 11c.

Figure 10 also illustrates RAO values for partiallynonlinear simulations using adaptive latching control, where the improvement of the adaptive strategy compared to the fixed-time latching strategy is seen to be significant.

Excitation and static FK forces, using the adaptive latching strategy, are shown in Fig. 12. Figure $12 \mathrm{~b}$ shows the static FK forces, where the shape of the linear and partiallynonlinear curves are rather similar, while the amplitude is lower for the partially-nonlinear case. In contrast, it can be seen in Fig. 12a that, even though the amplitude of linear and partially-nonlinear simulations is similar, their dynamics are different.

The comparison of the linear and partially-nonlinear forces can be extended to all the range of incident waves by comparing the amplitude of the static and dynamic FK forces for each incident wave. Taking the linear amplitude as a reference, partially-nonlinear forces are normalized to

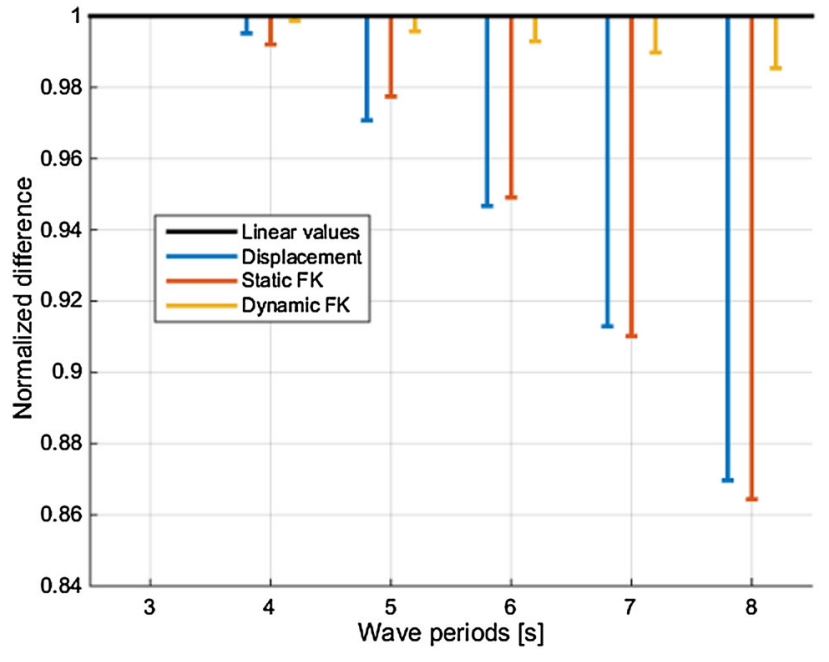

Fig. 13 Normalized differences between the linear and partiallynonlinear displacement, static FK force and excitation force amplitudes for all the analysed wave periods, where 1 corresponds to the linear case

analyse the impact of each force. In addition, the displacement of the device can also be normalized against the linear case. Figure 13 shows normalized differences between linear and partially-nonlinear displacement, static FK force and excitation force, where the value 1 corresponds to the linear case. The results from the partially-nonlinear model are always lower, which is to be expected, and the difference increases for all the three variables as waves become longer and higher.

However, while the excitation force for the partiallynonlinear case is always very close to the linear case, the difference between the linear and partially-nonlinear static FK forces is significant. Furthermore, one can observe in Fig. 13 that differences between the linear and partiallynonlinear cases are very similar for the displacement and the static FK forces, which demonstrates the higher relevance of the static part.

Power production assessment for the cylinder and the sphere, based on linear and partially-nonlinear simulation models, is calculated and illustrated in Fig. 14a, b, respectively. For the cylinder, almost identical results are obtained from both simulation models. In the case of the sphere, although the device motion amplitude increases substantially in the partially-nonlinear model simulations using the adaptive latching strategy, such motion never reaches the values of the linear case. Therefore, linear model simulations appear to overestimate the device motions and, consequently, the power absorption, as shown in Fig. 14b.

\section{Discussion}

Using nonlinear waves theory for the implementation of the nonlinear FK forces is theoretically more consistent, as 


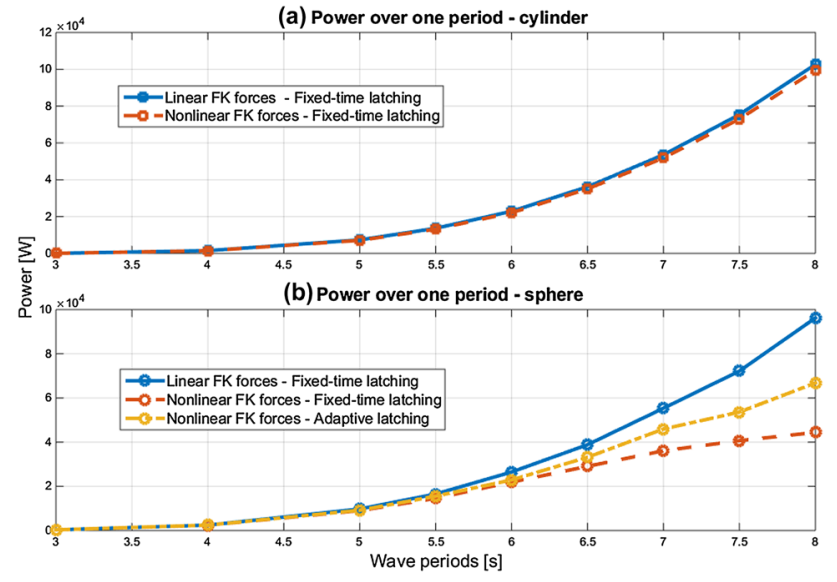

Fig. 14 Power production assessment for the cylinder (above) using only the fixed-time latching strategy and the sphere (below) using the fixed-time and adaptive latching strategies, for linear and partiallynonlinear simulation models

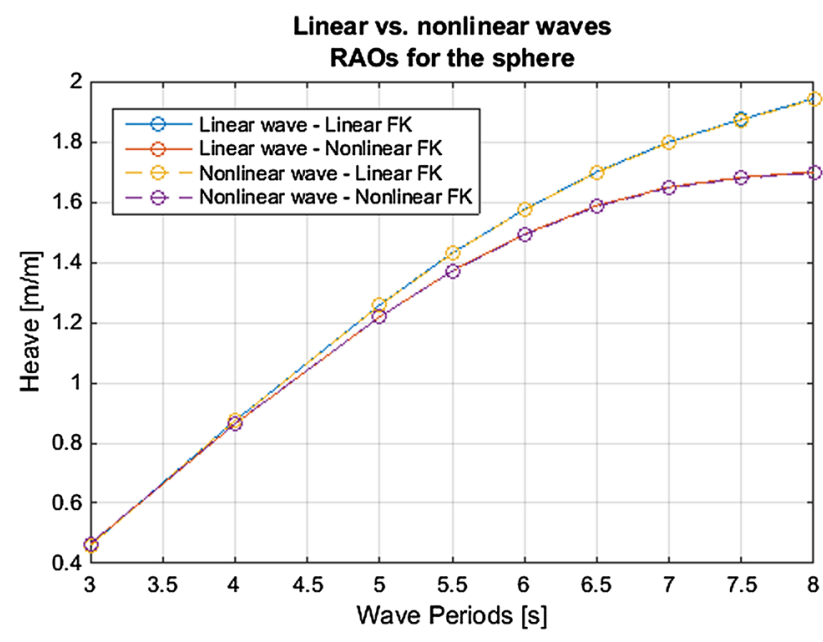

Fig. 15 Comparison of the RAOs for the sphere, using linear and nonlinear waves and linear and nonlinear FK forces

mentioned in Sect. 3.2, due to the nonlinear boundary condition on the free-surface $(P(z=\eta)=0)$. However, comparing the RAOs of the sphere from the present paper using the nonlinear wave theory to the RAOs from Penalba et al. (2015) using Airy's theory (linear), one can note that the difference is imperceptible, as illustrated in Fig. 15. Therefore, for low order nonlinear waves, the approximation using the linear wave theory seems to be appropriate even with nonlinear FK forces. For higher order nonlinear waves (steeper waves), higher differences are to be expected between results using linear and nonlinear wave theories, but further research is necessary to confirm that tendency. Notwithstanding, the waves within the power production region tend to be linear or low order nonlinear, as Penalba et al. (2017) suggests based on the power production data of different real devices during open-ocean tests. Therefore, higher order nonlinear waves may not be necessary within the power production region.
Regarding the relevance of nonlinear FK forces, Fig. 7 shows that there is little additional value in the incorporation of nonlinear FK forces in the simulation model for the cylinder. While this observation is limited to the device geometry and wave (and control) excitation examined, it suggests that nonlinear FK forces are not especially relevant in mathematical models for devices with a constant CSA and a single degree of freedom in heave, provided that the device is deployed in deep-water. In contrast, Fig. 10 shows a marked difference between linear and nonlinear RAOs for fixed-time latching control in the case of the sphere, which has a nonuniform CSA.

Clearly, from Figs. 10 and 11, the fixed-time latching strategy is inadequate for the case of the device with a nonuniform CSA, i.e. the sphere. This shows the danger of using a linear WEC model for development of the control strategy though, in the case of latching, the control 'parameters' are only coarsely dependent on the WEC model. For more highly-tuned controllers, such as optimal numerical control (Bacelli and Ringwood 2015), the mismatch between a controller designed on a linear WEC model and the real WEC model behaviour is likely to be more significant. Further supporting evidence is provided in (Ringwood et al. 2015) and in (Kracht 2013), where a highly-tuned model-predictive controller failed to outperform a simple (but more robust to modelling errors) WEC controller.

Figure $14 \mathrm{~b}$ shows the danger in the use of linear FK models for power production assessment, where the WEC is under controller conditions. The linear model overestimates the power production, due to the overestimation of the displacement, which gives erroneous indications of the economic viability of a controlled WEC topology.

In summary, there are three separate issues which are highlighted by the use of nonlinear FK modelling for devices with a non-uniform CSA under control:

- Simulation of WEC device motion under control,

- The use of WEC models for control design, and

- The assessment of power production capability of WECs under controlled conditions.

The common factor in the three applications listed above is that the device is under controlled conditions and is, therefore, subject to direct control forces which may have significantly greater frequency content than would issue from wave excitation alone. In addition, the timing interactions between wave excitation and control forces can also serve to generate exaggerated motion and higher device/fluid relative velocities. There is a significant number of documented studies where linear Froude-Krylov force models have been shown to well describe WEC motions under wave excitation alone e.g. (Paparella et al. 2015; Garcia-Rosa et al. 2015). However, more recently, some studies under control 
excitation (Ringwood et al. 2015; Giorgi and Ringwood 2015) (including the study described in this paper) suggest that linear models may not be adequate in such a case.

\section{Conclusion}

The present paper proves the suitability of linear wave theories for low order nonlinear waves, even with nonlinear FK forces, despite the lack of consistency on the boundary conditions. In addition, the paper demonstrates the value of including nonlinear Froude-Krylov forces in modelling generic device shapes for wave energy applications in the presence of regular waves. The significant difference in the results for the cylinder and sphere shows the influence of non-uniform cross-sectional area on the need for nonlinear FK force modelling. It is to be expected that the more substantial the gradient of the CSA is, the greater the need for nonlinear FK forces is. However, most importantly, the paper shows the importance of including realistic control force signals for the simulation and power production assessment of WECs under control, and as a platform for model-based control design, regardless of the implemented control strategy. In general, hydrodynamic modelling of WECs (and the subsequent validation of such models) is normally performed in relation to the free response of a device to wave excitation or, at best, the inclusion of some linear PTO damping element, which always serves to dampen the WEC motion and is not considered here as a 'control strategy'. Various studies, including (Penalba et al. 2015) and (Ringwood et al. 2015), have shown that, under (even significant) wave excitation alone, there is little value in utilising a nonlinear hydrodynamic model for various device shapes.

Therefore, a judicious choice needs to be made in relation to the decision to incorporate nonlinear FK forces in WEC hydrodynamic models. The use of nonlinear FK forces incurs a not insignificant computational overhead, with approximately an order of magnitude difference in computation time between the linear and nonlinear FK models, mainly due to the need to recalculate the instantaneous wetted surface at each time step. Further work need to be performed to asses the influence of including nonlinear Froude-Krylov forces when irregular waves are considered.

Acknowledgements This material is based upon works supported by the Science Foundation Ireland under Grant No. 13/IA/1886 and the Marine and Renewable Energy Ireland (MaREI) centre under Grant No. $12 / \mathrm{RC} / 2302$.

\section{References}

Babarit A (2010) Achil3D v2.011 user manual. Laboratoire de Mécanique des Fluides CNRS, Ecole Central de Nantes
Babarit A, Clément A (2006) Optimal latching control of a wave energy device in regular and irregular waves. Appl Ocean Res 28(2):77-91

Babarit A, Delhommeau G (2015) Theoretical and numerical aspects of the open source BEM solver NEMOH. In: 11th European wave and tidal energy conference, Nantes

Babarit A, Laporte-Weywada P, Mouslim H, Clement AH (2009) On the numerical modelling of the nonlinear behaviour of a wave energy converter. In: Proceedings of the ASME 28th international conference on ocean, offshore and arctic engineering, Honolulu, OMAE, vol 4, pp 1045-1053. doi:10.1115/OMAE2009-79909

Bacelli G, Ringwood JV (2015) Numerical optimal control of wave energy converters. IEEE Trans Sustain Energy 6(2):294-302

Costa PR, Garcia-Rosa PB, Estefen SF (2010) Phase control strategy for a wave energy hyperbaric converter. Ocean Eng 37(1718):14831490. doi:10.1016/j.oceaneng.2010.07.007

Cretel J, Lewis A, Lightbody G, Thomas G (2010) An application of model predictive control to a wave energy point absorber. Control Methodol Technol Energy Effic 1:267-272

Cummins W (1962) The impulse response function and ship motions. Schiffstechnik 9(Heft 47):101-109

Delhommeau G (1993) Seakeeping codes AQUADYN and AQUAPLUS. In: Proceedings of the 19th WEGEMT school on numerical simulation of hydrodynamics: ships and offshore structures, Nantes

Fenton J (1990) Nonlinear wave theories. The Sea 9(1):3-25

Fusco F, Ringwood J (2010) Short-term wave forecasting with ar models in real-time optimal control of wave energy converters. In: 2010 IEEE international symposium on industrial electronics (ISIE), pp 2475-2480. doi:10.1109/ISIE.2010.5637714

Garcia-Rosa P, Costello R, Dias F, Ringwood JV (2015) Hydrodynamic modelling competition: Overview and approaches. In: International conference on offshore mechanics and arctic engineering. Ocean renewable Energy, vol 9. doi:10.1115/OMAE2015-42182

Gilloteaux JC (2007) Mouvements de grande amplitude d'un corps flottant en fluide parfait. Application a la recuperation de l'energie des vagues. $\mathrm{PhD}$ thesis, Ecole Centrale de Nantes (ECN)

Gilloteaux JC, Bacelli G, Ringwood J (2008) A nonlinear potential model to predict large-amplitude-motions: application to a multibody wave energy converter. In: Proceedings of the 10th world renewable energy conference, Glasgow

Giorgi G, Ringwood J (2015) Implementation of latching control in a numerical wave tank. J Eng Mar Energy. Preprint version

Giorgi G, Penalba M, Ringwood J (2016) Nonlinear hydrodynamic force relevance for heaving point absorber and oscillating surge converters. In: Proceedings of the Asian wave and tidal energy conference, Singapore

Hals J, Bjarte-Larsson T, Falnes J (2002) Optimum reactive control and control by latching of a wave-absorbing semisubmerged heaving sphere. In: ASME 2002 21st international conference on offshore mechanics and arctic engineering. American Society of Mechanical Engineers, New York, pp 415-423

Hals J, Ásgeirsson GS, Hjálmarsson E, Maillet J, Möller P, Pires P, Guérinel M, Lopes M (2016) Tank testing of an inherently phasecontrolled wave energy converter. Int J Mar Energy 15:68-84

Kracht P (2013) Wave prediction and its implementation on control systems of wave-energy converters. EU MaRINet infrastructure access report, Frauenhofer IWES

Lawson M, Yu YH, Nelessen A, Ruehl K, Michelen C (2014) Implementing nonlinear buoyancy and excitation forces in the WECSIM wave energy converter modeling tool. In: ASME 2014 33rd international conference on ocean, offshore and arctic engineering. American Society of Mechanical Engineers, New York, pp V09BT09A043-V09BT09A043

Merigaud A, Gilloteaux J, Ringwood J (2012) A nonlinear extension for linear boundary element method in wave energy device modelling. 
In: Proceedings of the 31 st international conference on ocean, offshore and arctic engineering (OMAE), Rio de Janeiro, pp 615-621

Paparella F, Bacelli G, Mícheál O, Ringwood J (2015) On the solution of multi-body wave energy converter motions using pseudo-spectral methods. In: Proceedings of European wave and tidal energy conference, Nantes

Penalba M, Mérigaud A, Gilloteaux JC, Ringwood JV (2015) Nonlinear froude-Krylov force modelling for two heaving wave energy point absorbers. In: Proceedings of European wave and tidal energy conference, Nantes

Penalba M, Giorgi G, Ringwood J V (2017) Mathematical modelling of wave energy converters: a review of nonlinear approaches. Renew Sustain Energy Rev 78:1188-1207. doi:10.1016/j.rser.2016.11. 137
Rienecker M, Fenton J (1981) A fourier approximation method for steady water waves. J Fluid Mech 104:119-137

Ringwood JV, Butler S (2004) Optimisation of a wave energy converter. In: Proceedings of the IFAC conference on control application sin marine Systems, Ancona, Italy

Ringwood JV, Davidson J, Giorgi S (2015) Optimising numerical wave tank tests for the parametric identification of wave energy device models. In: Proceedings of the 34th international conference on ocean, offshore and arctic engineering (OMAE 2015)

WAMIT Inc M (2013) WAMIT v7.0 manual

Zurkinden A, Ferri F, Beatty S, Kofoed J, Kramer M (2014) Nonlinear numerical modelling and experimental testing of a point-absorber wave energy converter. Ocean Eng 78:11-21 